\title{
Effect of force and location of bottleneck for particle moving through window under encapsulation
}

\author{
ANIRBAN SHARMA and PRADIP KR. GHORAI* \\ Department of Chemical Sciences, Indian Institute of Science Education and Research Kolkata, \\ Mohanpur, West Bengal 741 246, India \\ E-mail: pradip.ghorai@gmail.com
}

MS received 5 April 2017; revised 2 June 2017; accepted 4 June 2017

\begin{abstract}
One of the central assumptions to explain appearance of an anomalous diffusion regime in microporous materials is the cancellation of forces experienced by a moving particle at the bottleneck. In this work, we examine this issue of force cancellation inside a microporous material exploring the diffusion path and cross-checking bottleneck associated with diffusion. Conventional wisdom is that window of a microporous material is the bottleneck for diffusion. Our study reveals that bottleneck for diffusion is not at the window, it is always $2.6 \AA$ to $3.0 \AA$ away from the window plane and depending on guest size, it lies in different parts of the inner surface of $\alpha$-cage. Window has significant effect in diffusion process only when diffusing particle size is comparable or larger than the window free diameter. Window does not play any role if particle size is smaller than window free diameter. We show that in the anomalous diffusion regime there is no signature of force cancellation at the window. Activation energy and total force along the diffusion path are responsible for the existence of anomalous diffusion regime in microporous materials.
\end{abstract}

Keywords. Force cancellation; anomalous regime; encapsulation; microporous materials; molecular dynamics simulation.

\section{Introduction}

Microporous materials have enormous potential of providing accurate and specific separation of gases. ${ }^{1,2}$ They are widely used to separate molecules of particular size and shape from their mixtures. ${ }^{3-5}$ Zeolites are one of the most important microporous materials in industrial separation and reaction processes. ${ }^{6} \mathrm{~A}$ wide variety of different zeolites are used in several applications such as catalysis and separation. ${ }^{7}$ In catalytic process, diffusion inside crystalline materials affects both the reactions and selectivities. ${ }^{8}$ Recently, it is observed that zeolites has potential for drug loading and delivery to the cancer cells. ${ }^{9,10}$ Self-diffusion of the drug inside host zeolite is an important phenomena for these type of drug delivery systems. ${ }^{11}$ Faujasite or zeolite $\mathrm{NaY}$ which is used as a shape selective catalyst in petrochemical industries, is an important class of microporous material with large surface area, well-defined window structure and high porosity. ${ }^{5}$ It is also used in the adsorption process and separation of hydrocarbons,

\footnotetext{
*For correspondence
}

aromatics, etc. For its wide range of applications, diffusion and adsorption studies of guests in this zeolite have become extremely important for both fundamentally and industrially. Different experimental techniques such as quasi-elastic neutron scattering and pulsed field gradient NMR, together with various computer simulations such as molecular dynamics (MD) and Monte Carlo (MC) methods have become powerful tools for studying structural and dynamical properties of guests inside host zeolites. ${ }^{12-17}$ Due to confinement of guest by host zeolite, unexpected dependencies of self - diffusivity is observed. Yashonath and Santikary first observed diffusion anomaly in zeolite $\mathrm{NaY}$ and $\mathrm{NaCaA}^{18}$ where self-diffusion coefficient $(D)$ increases with particle size $\left(\sigma_{g g}\right)$. The diffusion maximum in microporous material was also observed by quasi-elastic neutron scattering (QENS) experiment. ${ }^{19,20}$ The observed diffusion maximum was explained in terms of force experienced by the guest at the window during intercage diffusion. ${ }^{21}$ Though it was explained that existence of diffusion maximum for an optimum $\sigma_{g g}$ is due to cancellation of forces at the window, ${ }^{22}$ it was not computed explicitly the amount of force experienced by a diffusing species only at the window. Chitra and Yashonath explained 
observed anomalous regime in connection with activation energy $\left(E_{a}\right){ }^{23}$ Though they observed minimum activation energy when diffusion is maximum, $E_{a}$ for other $\sigma_{g g}$ could not explain complete dependence of $D$ on $\sigma_{g g}$. To give an example, though $E_{a}$ is much lower for $\sigma_{g g}=6.8 \AA$ as compared to $\sigma_{g g}=2.67 \AA, D$ for $\sigma_{g g}=2.67 \AA$ is much higher than $\sigma_{g g}=6.8 \AA .{ }^{18}$ Though both $E_{a}$ and force are lower for bigger guest as compared to that for smaller particle, $D$ is much lower for the bigger guest. Later Yashonath and Chitra computed energy barrier at the window plane during intercage migration along minimum energy paths (MEPs) but those studies were at absolute zero temperature. At any finite temperature, diffusing guest might not migrate from one cage to another through MEPs. This study also counter intuitively showed that $E_{a}$ is large for the smaller guest which has large $D .{ }^{23}$ In this study, we explain that force cancellation at the window does not play any role in the diffusion process. We compute energy barrier, force and other related properties along the diffusion path to explain dependence of $D$ on $\sigma_{g g}$ in crystalline microporous materials.

\section{Methods}

\subsection{Structure of zeolite $\mathrm{NaY}$}

In this study we use faujasite zeolite (NaY) as microporous material which has the same framework as natural mineral faujasite, as shown by neutron diffraction studies reported by Fitch $e$ t al. ${ }^{24} \mathrm{NaY}$ belongs to the $F d \overline{3} m$ space group and room temperature phase has a cubic unit cell with cell length $24.8536 \AA$. The framework consists of a tetrahedral array of sodalite units interconnected through six membered oxygen bridges as shown in Figure 1(a). Ten sodalite units form a single large cage-like unit, known as $\alpha$-cage or supercage of diameter $\sim 11.8 \AA$. All supercages are connected to four neighbouring cages through 12-membered oxygen rings of diameter $\sim 7.5 \AA$ (Figure 1(b)). All simulations are carried out for unit cell composition of $\mathrm{Na}_{48} \mathrm{Si}_{144} \mathrm{Al}_{48} \mathrm{O}_{384}$ with $\mathrm{Si} / \mathrm{Al}$ ratio 3.0. The extra framework sites I and II are completely filled by $\mathrm{Na}^{+}$ions with no partial occupancy.

\subsection{Intermolecular potential functions}

Zeolite framework is considered as rigid that is the coordinates of zeolite atoms and extra framework cations are not included in the MD integration. Guest-guest as well as guest-zeolite interaction have been modeled in terms of (6-12) LennardJones (LJ) potential without any Coulomb interaction. The LJ potential for guest-guest interaction is given by

$\phi_{g g}\left(r_{g g}\right)=4 \epsilon_{g g}\left[\left(\frac{\sigma_{g g}}{r_{g g}}\right)^{12}-\left(\frac{\sigma_{g g}}{r_{g g}}\right)^{6}\right]$
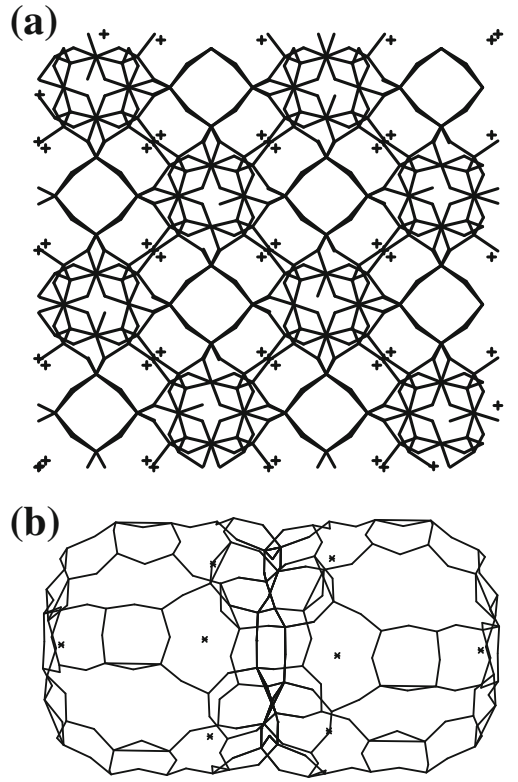

Figure 1. (a) One unit cell of zeolite $\mathrm{Y}$ and (b) two nearest $\alpha$-cages are interconnected through 12-ring window.

where subscript $g$ stands for the guest, $\epsilon_{g g}$ and $\sigma_{g g}$ are the well-depth and LJ diameter respectively.

The guest-host interaction is given by

$\phi_{g h}\left(r_{g h}\right)=4 \epsilon_{g h}\left[\left(\frac{\sigma_{g h}}{r_{g h}}\right)^{12}-\left(\frac{\sigma_{g h}}{r_{g h}}\right)^{6}\right]$

where subscript $h$ stands for the host atoms ( $h=O$ and $\mathrm{Na}$ ). $\mathrm{As} \mathrm{Si}$ and $\mathrm{Al}$ atoms of the host are surrounded by the bulkier oxygen, close approach of guest to $\mathrm{Si} / \mathrm{Al}$ atoms is not possible. For this reason, short-range interactions are included between the guest - $O$ and guest - $\mathrm{Na}$ only.

Total interaction energy between the guests is given by

$U_{g g}=\frac{1}{2} \sum_{g=1}^{N} \sum_{g=1}^{N} \phi_{g g}\left(r_{g g}\right)$

and total guest-host interaction is given as

$U_{g h}=\frac{1}{2} \sum_{g=1}^{N} \sum_{h=O, N a} \phi_{g h}\left(r_{g h}\right)$

where $N$ represents total number of guests. As zeolite structure is considered as rigid and model solutes are nonpolarizable, polarization interaction between the guest and host zeolite has been neglected. Previous studies showed that polarization interaction energy is about only few percent of the total energy and hence even though the polarization interaction has been neglected, it may not have large impact on self-diffusivity. ${ }^{18}$ 
Table 1. Intermolecular potential parameters for different atoms of the host zeolite.

\begin{tabular}{lcc}
\hline nature & $\epsilon(\mathrm{kJ} / \mathrm{mol})$ & $\sigma(\AA)$ \\
\hline $\mathrm{O}-\mathrm{O}$ & 1.2891340 & 2.544700 \\
$\mathrm{Na}-\mathrm{Na}$ & 0.0392347 & 3.369378 \\
\hline
\end{tabular}

\subsection{Computational details}

Molecular Dynamics simulations have been carried out in the microcanonical ensemble that is with fixed $N, V$ and $E$ where $N$ represents total number of guests, $V$ represents volume of the simulation cell and $E$ is total energy of the system. $4 \times$ $4 \times 4$ cubic unit cells of zeolite $\mathrm{NaY}$ is considered in all the simulations. We use concentration of 1 guest/cage with a total of 512 guests in the present study. Mass of the guest is taken as $131 \mathrm{amu}$. Integration of the equations of motion of the guest has been performed while zeolite atoms have been kept fixed during the whole simulations. We use an integration time step of $10 \mathrm{fs}$ which yields good conservation of energy and linear momentum. Cubic periodic boundary condition is applied in all three directions. A spherical cut-off of 12 $\AA$ is used for guest-guest as well as guest-host interaction. Equilibration is performed over a duration of $4.0 \mathrm{~ns}$. All the properties are computed from trajectories stored at an interval of $0.2 \mathrm{ps}$ from a production run of $25.0 \mathrm{~ns}$. All calculations are carried out at $150 \mathrm{~K}$. Different intermolecular potential parameters are listed in Table 1. Cross-interaction parameters are computed from the Lorentz-Berthleot combination rule. ${ }^{25}$ $\epsilon_{g g}$ for guest-guest interaction is taken as $1.837394 \mathrm{~kJ} / \mathrm{mole}$. We use the same potential as Yashonath and co-workers used previously. ${ }^{18}$

\section{Results and Discussions}

\subsection{Thermodynamics properties}

Guest-host interaction energy $\left(U_{g h}\right)$ and guest-guest interaction energy $\left(U_{g g}\right)$ computed at $150 \mathrm{~K}$ for different $\sigma_{g g}$ are shown in Figure 2(a) and 2(b) respectively. $U_{g h}$ and $U_{g g}$ are negative for all $\sigma_{g g}$ indicating interaction energies are attractive in nature. Variation of $U_{g h}$ with $\sigma_{g g}$ can be divided into three regimes; (i) regime I when $3.07 \AA \leq \sigma_{g g} \leq 4.96 \AA$, regime II when 4.96 $\AA<\sigma_{g g} \leq 6.0 \AA$ And regime III when $6.0 \AA<\sigma_{g g} \leq$ $6.80 \AA$. When $\sigma_{g g}$ is much smaller than window diameter $\left(\sigma_{w w}\right), U_{g h}$ decreases very slowly with the guest size and when $\sigma_{g g} \approx \sigma_{w w}, U_{g h}$ decreases rapidly. In the anomalous regime, that is in regime II, decrease in $U_{g h}$ with $\sigma_{g g}$ is moderate. Guest-guest interaction is very less as compared to guest-zeolite interaction. Initially

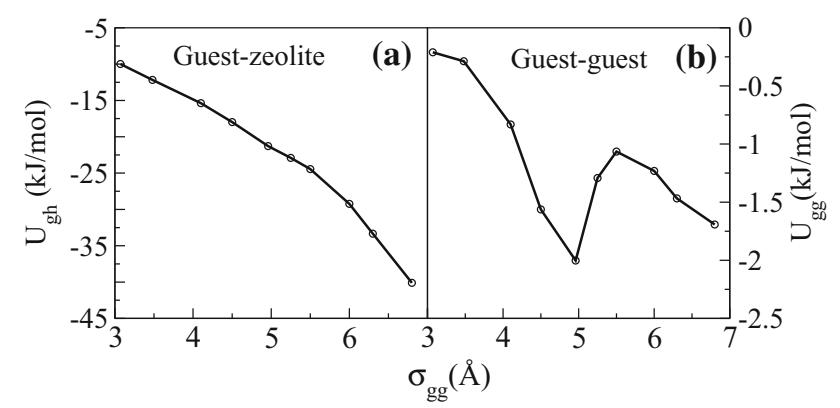

Figure 2. (a) Guest - zeolite and (b) guest - guest interaction energy for different $\sigma_{g g}$.

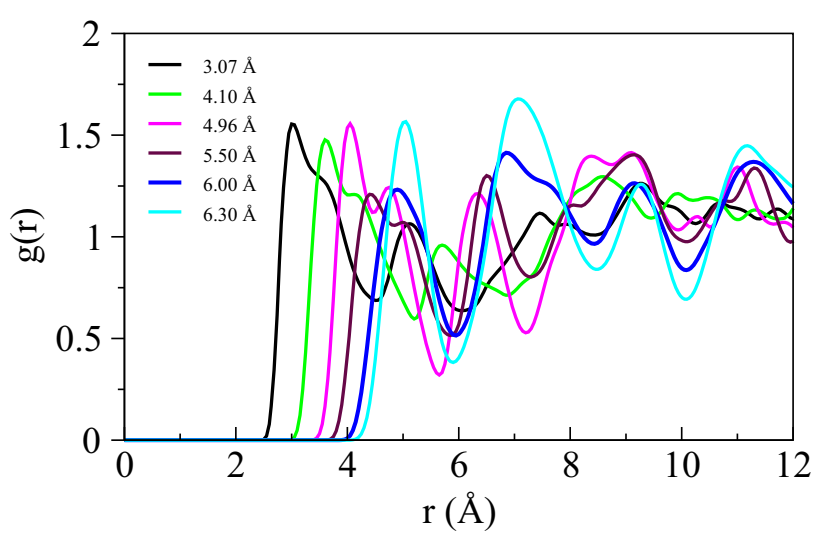

Figure 3. RDF's between the guest and oxygen atom of the zeolite for different $\sigma_{g g}$ at $\mathrm{T}=150 \mathrm{~K}$.

$U_{g g}$ decreases with $\sigma_{g g}$ and then increases in the anomalous regime and again decreases when $\sigma_{g g} \approx \sigma_{w w}$. This clearly shows that though $U_{g g}$ is very small as compared to $U_{g h}$, it has imperceptible effect to increase $D$ in the anomalous regime.

\subsection{Structure and energetics}

Radial distribution functions (RDF's) between the guest and oxygen atom of host zeolite are shown in Figure 3 for different $\sigma_{g g}$. In all cases, RDF's exhibit welldefined peaks suggesting guests are largely localized to certain well-defined regions within the $\alpha$-cages of $\mathrm{NaY}$. As distance between guest and host affects peak height in RDF's, thus dynamics of the diffusion species, we particularly put importance on the first two peaks in Figure 3. Intensity and broadening of the peaks strongly depend on $\sigma_{g g}$. When $\sigma_{g g}<4.96 \AA$, intensity of the first peak height decreases and the diffuse second peak becomes more prominent with $\sigma_{g g}$. Both the peaks height are maximum for $\sigma_{g g}=4.96 \AA$. When $\sigma_{g g}>4.96 \AA$, both peak heights decrease and second peak completely vanishes for $\sigma_{g g}>6.0 \AA$. When $\sigma_{g g}>6.0 \AA$, first peak intensity again increases rapidly and reaches to a maximum for 


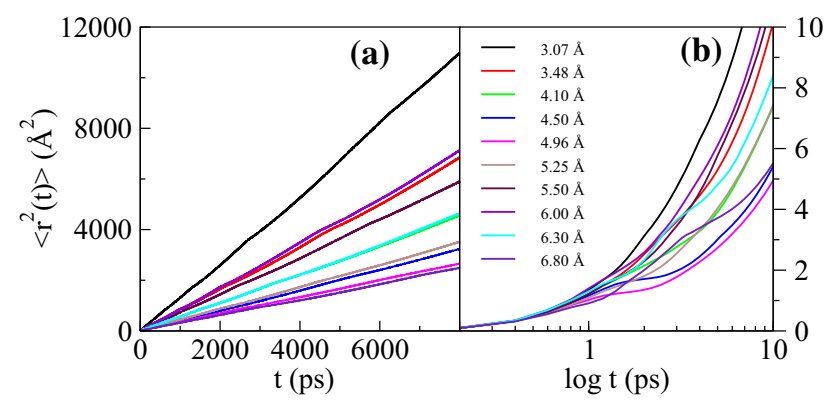

Figure 4. Mean squared displacement (MSD) for different $\sigma_{g g}$ for (a) long and (b) short time.

the largest $\sigma_{g g}$. It indicates that depending on $\sigma_{g g}$, diffusing species evolve from a more ordered packing to disordered packing. This certainly has some effect on the dynamical properties which are discussed in details in the later section.

\subsection{Dynamical properties}

3.3a Diffusivity: Figure 4 (a) shows mean squared displacement (MSD), $<r^{2}(t)>$ up to $8.0 \mathrm{~ns}$ and Figure 4(b) shows short time behaviour of the MSD for different $\sigma_{g g}$. The linear relationship between $\left\langle r^{2}(t)>\right.$ vs $t$ at large $t$ suggests that the motion is diffusive. For $\sigma_{g g}=4.5 \AA$ and $4.96 \AA$, a sub-diffusive region is seen whereas for very small and large $\sigma_{g g}$, we do not observe any sub-diffusive regime. Figure 4(b) shows two different regimes, (i) for $3.07 \AA \leq \sigma_{g g}<3.48 \AA$; ballistic regime where $<r^{2}(t)>\propto t^{2}$ for $t<1.4$ ps and (ii) a diffusive regime where $\left\langle r^{2}(t)>\propto t\right.$ for $t>1.4$ ps. As $\sigma_{g g}$ increases, a plateau or cage regime appears, where diffusing species find themselves trapped inside $\alpha$-cage. The plateau regime is more intense for $\sigma_{g g}=$ $4.96 \AA$ for which MSD is minimum and this plateau regime decreases and completely vanishes for $\sigma_{g g}=6.0$ $\AA$ for which MSD is maximum. When $\sigma_{g g}>6.0 \AA$, two plateau regimes are seen and diffusive regimes start only when $t>10 \mathrm{ps}$. The self-diffusion coefficient is computed from the Einstein relation ${ }^{25}$ as follow;

$D=\lim _{t \rightarrow \infty} \frac{\left\langle r^{2}(t)\right\rangle}{2 d t}$

where $d$ is the dimensionality of the system (in the present case $d=3$ ) and $t$ is the time.

Figure 5 shows $D$ as a function of $1 / \sigma_{g g}^{2}$. We observe two different regime: (i) linear regime where $D$ decreases with $1 / \sigma_{g g}^{2}$ and (ii) anomalous regime where $D$ increases with $1 / \sigma_{g g}^{2}$. We also observe a diffusion maximum for $\sigma_{g g}=6.0 \AA$ and diffusion minimum for $\sigma_{g g}$ $=4.96 \AA$. To understand the existence of anomalous regime, we compute distribution of guest, $P\left(r_{c w}\right)$ at the

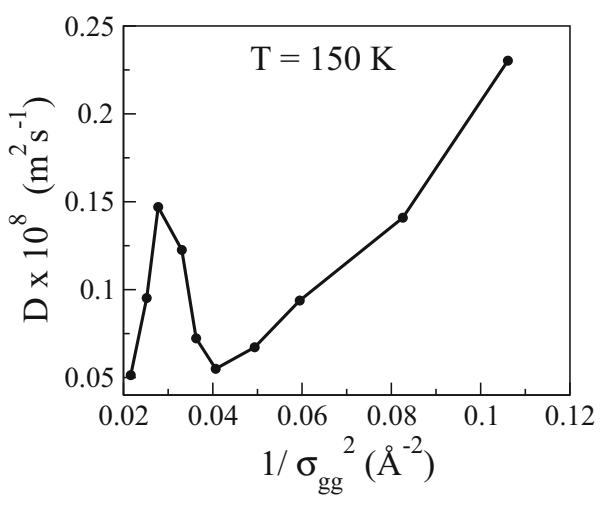

Figure 5. Self diffusion coefficient as a function of $1 / \sigma_{g g}^{2}$.

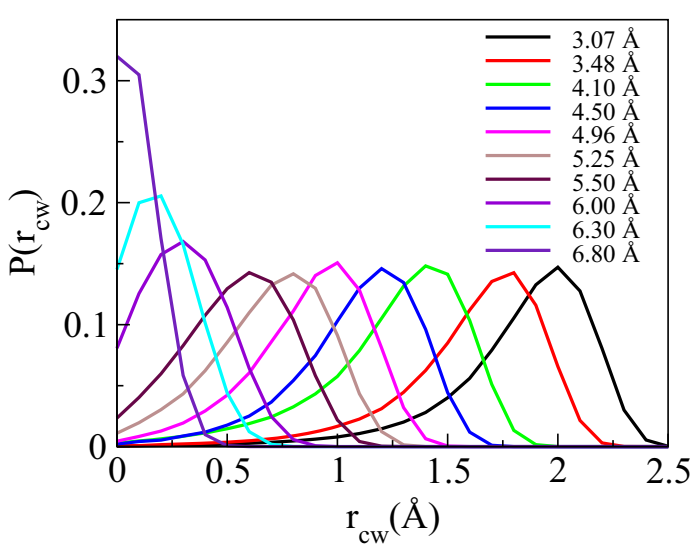

Figure 6. Distribution of guest as a function of $c w$ for different $\sigma_{g g}$.

window plane as a function of $r_{c w}$ (Figure 6). $r_{c w}$ is the distance of a guest from the window center when guest lies in the window plane during intercage migration. Except $\sigma_{g g}=6.8 \AA$, we observe distinct peak in all cases for non zero value of $\mathrm{r}_{c w}$. This implies that during diffusion through window, particle does not pass through window center. The preference of crossing towards window center increases with $\sigma_{g g}$. It was reported previously that when diffusing species pass through window, net force on diffusing particle decreases due to cancellation of forces at the window. ${ }^{18}$ As peak position in the $P\left(r_{c w}\right)$ decreases with $\sigma_{g g}$, if cancellation of force takes place, then $D$ should increase with $\sigma_{g g}$. But we find $D$ initially decreases in the linear regime, then increases in the anomalous regime and again decreases.

To identify the diffusion path of moving particle, we compute distribution of guest $[P(R)]$ on different planes which are perpendicular to the line joining a window center and corresponding $\alpha$-cage center. First we identify all the time steps when diffusing particle passes through the window. Then we consider 4 ps before and 


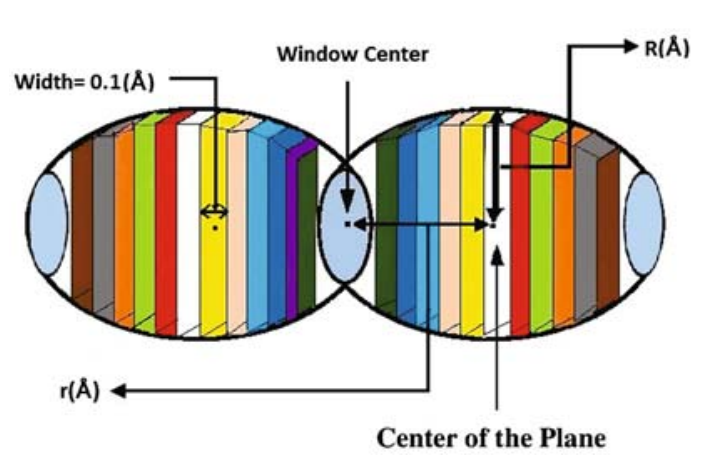

Figure 7. Schematic diagram of cage and window of zeolite NaY. $R$ represents the distance of a guest from the center of a particular plane and $r$ is the perpendicular distance of the a from window center.

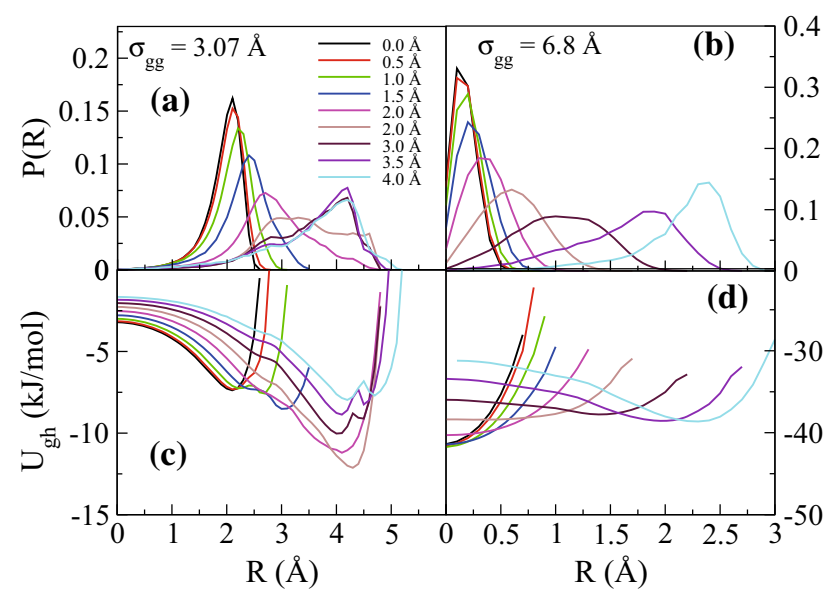

Figure 8. $P(r)$ for different planes for (a) $\sigma_{g g}=3.07 \AA$ and (b) $\sigma_{g g}=6.8 \AA$. (c) and (d) are the corresponding $U_{g h}$ for two different $\sigma_{g g}$.

after intercage crossing to find out locations of the diffusing particle and bin all these positions to different planes of width $0.5 \AA$. $R$ is the distance of a particle from the center of a particular plane and $r$ represents perpendicular distance of the planes from its nearest window (Figure 7). Plane at $r=0$ is the window plane and then moves toward $\alpha$-cage center. We compute $P(R)$ on each of these planes separately. Figure 8(a) and 8(b) show $P(R)$ as a function of $R$ for $\sigma_{g g}=3.07 \AA$ and $6.8 \AA$ respectively. Figure 8(c) and 8(d) show corresponding guest-host interaction energy. For both sizes, peak position in the distribution is seen at a point where $U_{g h}$ is minimum. Hence during diffusion process, location of the guest is mainly governed only by the guest-host interaction. Peak position in the distribution shifts towards large value of $R$ suggesting preferential position of the diffusing species towards inner surface of the $\alpha$-cage as it moves towards cage center. Due to availability of more space near cage center, width of $P(R)$ increases close to

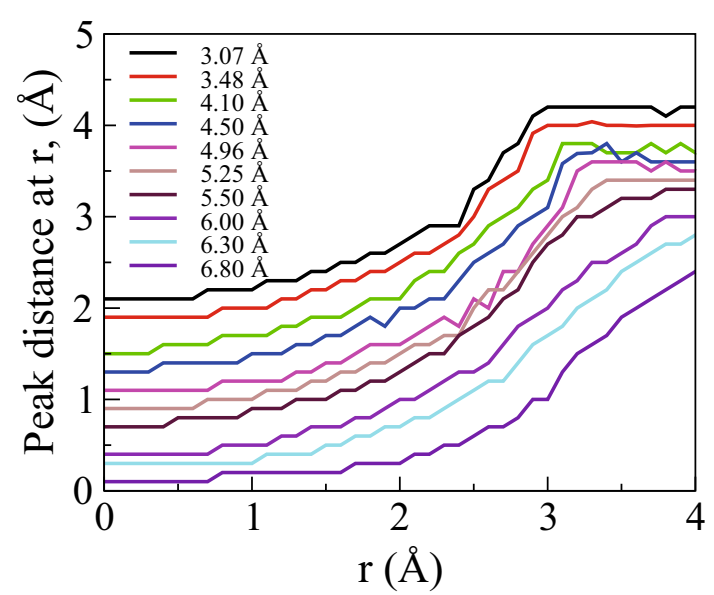

Figure 9. Peak positions in $P(R)$ as a function of $r$ for different $\sigma_{g g}$.

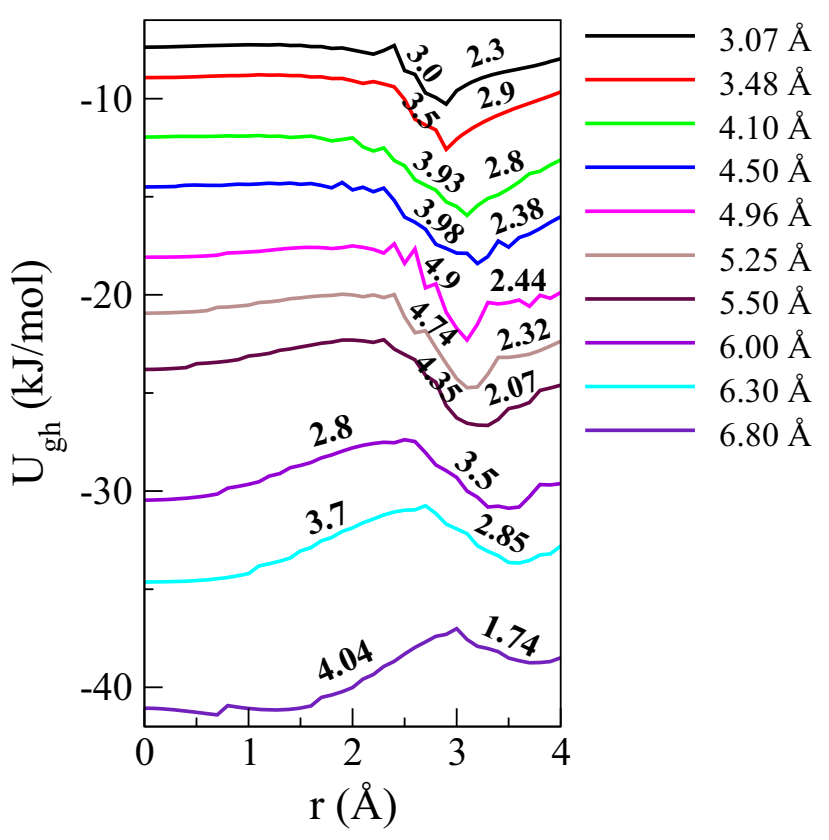

Figure 10. $U_{g h}$ along diffusion the path as a function of $r$ for different $\sigma_{g g}$. Bold numbers are $E_{a 1}$ and $E_{a 2}$ for different $\sigma_{g g}$.

the cage center than at the window. We compute $P(R)$ for all $\sigma_{g g}$ and identify peak position in $P(R)$ for different planes and join all the peak positions through a line. For any particular $\sigma_{g g}$, the line which connects all the peak positions in $P(R)$ is the most probable diffusion path through the window. Figure 9 shows diffusion path for different $\sigma_{g g}$ as a function of $r$. At the window plane $(r=0)$, particle diffuses closer to the window center as $\sigma_{g g}$ increases. For all $\sigma_{g g}$, particle moves along the inner surface of $\alpha$-cage. This suggests that diffusion process in nanopore is the surface mediated diffusion.

We now compute potential energy landscape along each diffusion path for different $\sigma_{g g}$. Figure 10 shows 


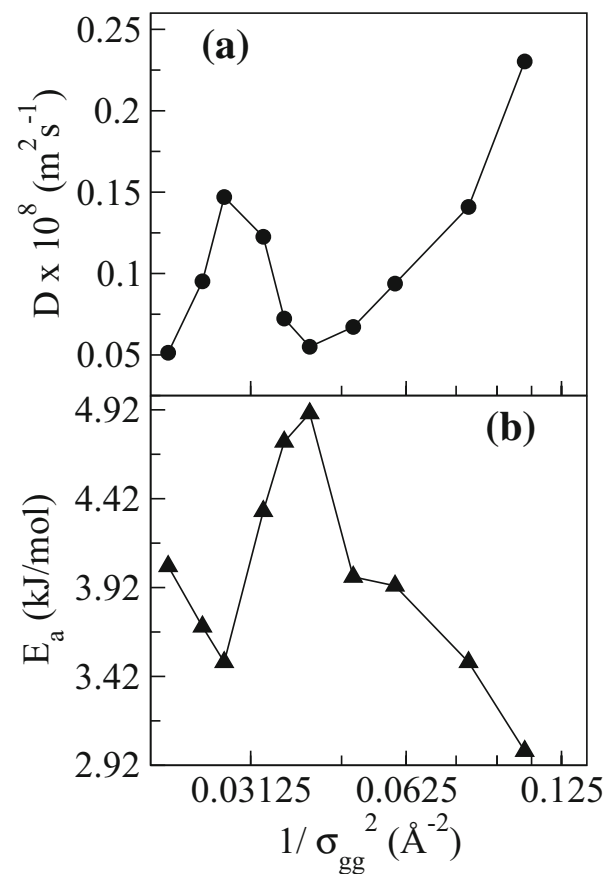

Figure 11. (a) $D$ as a function of $1 / \sigma_{g g}^{2}$ and (b) $E_{a}$ for different $\sigma_{g g}$ along the diffusion path

$U_{g h}$ as a function of $r$ along the diffusion path. Surprisingly, energy barrier is not seen at the bottleneck for all the particles. When $\sigma_{g g}<4.96 \AA, U_{g h}$ near window $(r \leq 2.0 \AA)$ is flat suggesting no energy barrier at the window which is known as the bottleneck for diffusion in microporous materials. For $\sigma_{g g} \geq 4.96 \AA$, $\sigma_{g g}$ exhibits a minimum near window and very close to the window, that is when $r \leq 1.0 \AA$, energy landscape is flat. For all $\sigma_{g g}$, two energy barriers are seen and their locations depend on $\sigma_{g g} . E_{a 1}$ is the energy difference between $r=0 \AA$ and $3.0 \AA$ ( $r$ varies from $2.6 \AA$ to 3.0 $\AA$ depending on $\sigma_{g g}$ ) and $E_{a 2}$ is the energy difference between $r=3.0 \AA$ (this varies from $2.6 \AA$ to $3.0 \AA$ depending on $\sigma_{g g}$ ) and $r=4.0 \AA$. For any $\sigma_{g g}$, maximum value between $E_{a 1}$ and $E_{a 2}$ is the energy barrier or activation energy for diffusion. When $\sigma_{g g} \leq 6.0 \AA$ one barrier is observed near $\alpha$-cage center $(r=4.0 \AA)$ and another is at $r \approx 2.5 \AA$. As $\sigma_{g g}$ increases $\left(\sigma_{g g}>6.0 \AA\right)$, energy barrier near cage center becomes insignificant and both energy barriers are seen at $r \approx 3.0 \AA$. When $\sigma_{g g}$ is small, significant energy barrier is seen near cage center which is unexpected and it decreases for large $\sigma_{g g}$. We also observe that barrier location moves marginally towards cage center as $\sigma_{g g}$ increases. It is clear from Figure 11 that nonmonotonic dependence of $D$ on $\sigma_{g g}$ is directly related to $E_{a}$. For $\sigma_{g g}=6.0 \AA E_{a}$ is minimum for which $D$ is maximum. Previously it was observed that though $D$ is extremely large for $\sigma_{g g}=3.07 \AA$ than
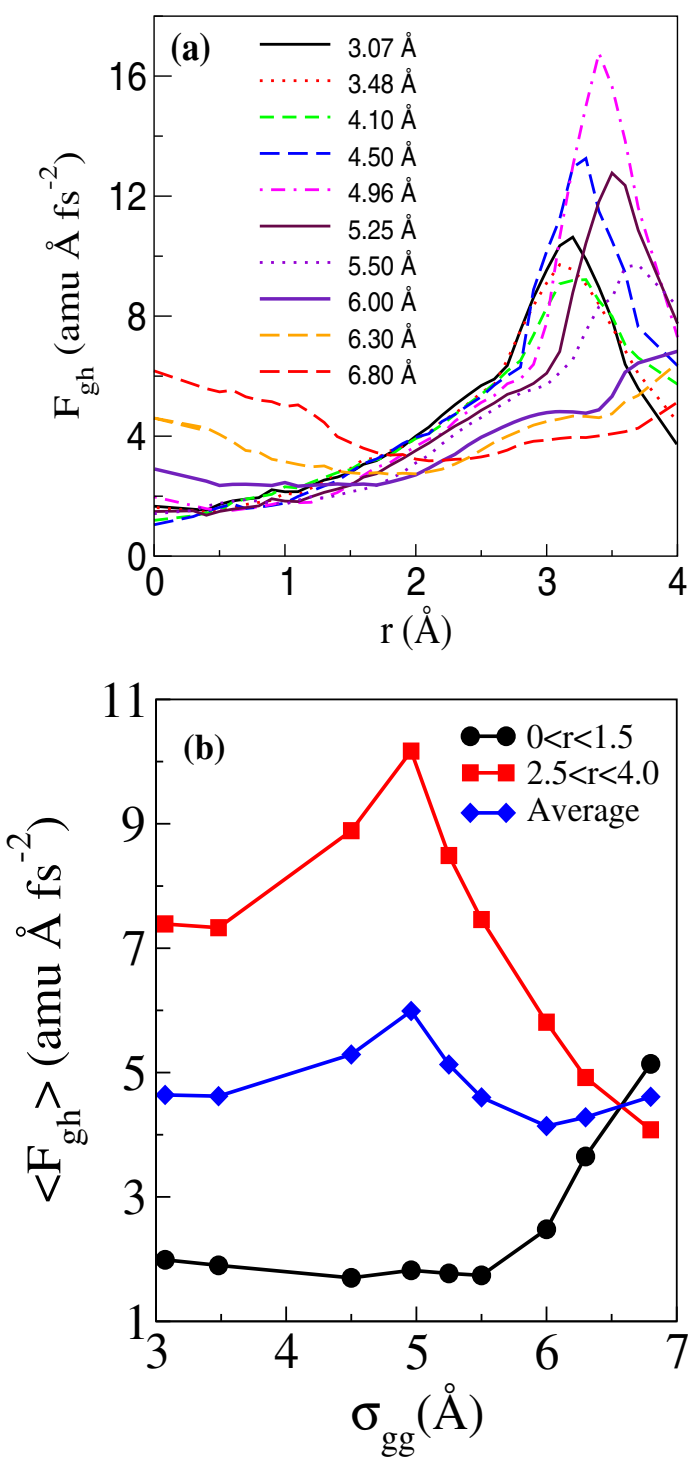

Figure 12. (a) $F_{g h}$ along diffusion path $r$ and (b) $\left\langle F_{g h}>\right.$ in two different regimes along the diffusion path for different $\sigma_{g g}$.

$\sigma_{g g}=6.0 \AA, E_{a}$ for $\sigma_{g g}=3.07 \AA$ is less than $\sigma_{g g}=6.0$ $\AA$ which is counter intuitive. ${ }^{26}$ They also reported very small $E_{a}$ for $\sigma_{g g}=6.8 \AA$ for which $D$ is nearly zero. As we compute $E_{a}$ along the diffusion path, quantitative agreement between $E_{a}$ and $D$ for all $\sigma_{g g}$ is observed previously.

In order to explore conventional wisdom of force $\left(F_{g h}\right)$ on $D$ at the window, we compute $F_{g h}$ on diffusing species along the diffusion path. Figure 12(a) shows $F_{g h}$ for different $\sigma_{g g}$. When $\sigma_{g g} \leq 5.5 \AA, F_{g h}$ is less and almost similar for all $\sigma_{g g}$ near window $(r=0)$. When $\sigma_{g g}=4.96 \AA$, for which $D$ is minimum, $F_{g h}$ at $r=0$ $\AA$ is minimum and $r=3.4 \AA$ it is maximum. When $\sigma_{g g}>4.96 \AA, F_{g h}$ near window increases and decreases away from the window. When $\sigma_{g g}=6.0 \AA$ for which $D$ 
Table 2. Residence time $\left(\tau_{r}\right)$ and intercage jump for different $\sigma_{g g}$.

\begin{tabular}{cccccc}
\hline$\sigma_{g g}(\AA)$ & \multicolumn{2}{c}{ Residence time $\left(\tau_{r}\right)(\mathrm{ps})$} & & \multicolumn{2}{l}{ Intercage jump /guest/sec. } \\
\cline { 2 - 3 } \cline { 5 - 6 } & Cage & Window & & $k_{c} \times 10^{-10}$ & $k_{v} \times 10^{-10}$ \\
\hline 3.07 & 44 & 1.6 & 0.311 & 0.132 \\
3.48 & 71 & 1.6 & 0.237 & 0.089 \\
4.10 & 100 & 1.6 & 0.172 & 0.062 \\
4.50 & 146 & 1.8 & & 0.102 & 0.042 \\
4.96 & 218 & 5.1 & 0.080 & 0.035 \\
5.25 & 117 & 6.6 & 0.154 & 0.054 \\
5.50 & 60 & 7.4 & 0.370 & 0.096 \\
6.00 & 51 & 13 & 1.523 & 0.115 \\
6.30 & 30 & 44 & 2.130 & 0.081 \\
6.80 & 28 & 84 & 2.981 & 0.021 \\
\hline
\end{tabular}

is maximum in the anomalous regime, $F_{g h}$ at the window is more than $\sigma_{g g} \leq 5.50 \AA$. Our study reveals no signature of force cancellation at the window for an optimum size of $\sigma_{g g}$ which was assumed previously. ${ }^{18,27}$ For $\sigma_{g g} \geq 6.0 \AA, F_{g h}$ increases significantly at the window and decreases near cage center. For $\sigma_{g g}=6.8 \AA, F_{g h}$ is maximum at the window plane whereas it is minimum away from the window plane. When $\sigma_{g g}$ is larger than window free diameter, $F_{g h}$ near window plays an important role for diffusion, whereas for $\sigma_{g g}<6.0 \AA$, $F_{g h}$ near $\alpha$-cage center has no significant effect on $D$. We also compute average total force $\left(<F_{g h}>\right)$ exerted on a diffusing species when particle is very close to the window $(0 \AA<r<1.5 \AA)$ and close to the $\alpha$-cage center $(2.5 \AA<r<4.0 \AA)$ along the diffusion path which are shown in Figure 12(b). When $\sigma_{g g} \leq 5.5 \AA$, close to the window $\left\langle F_{g h}>\right.$ is comparable for all $\sigma_{g g}$ and beyond $\sigma_{g g}>5.5 \AA$, it increases significantly. Even for average force, we do not observe any minimum near window for $\sigma_{g g}=6.0 \AA$ for which $D$ is maximum (Figure 5). Conventional wisdom of cancellation of forces at the window has no role for the existence of anomalous diffusion in microporous materials. In fact unless $\sigma_{g g}$ is larger than $\sigma_{w w}$, window does not play any significant role in the diffusion process. We further observe that away from the window, $<F_{g h}>$ increases with $\sigma_{g g}$ and it is maximum for $\sigma_{g g}=4.96 \AA$ and then decreases. Dependence of total $<F_{g h}>$ on $\sigma_{g g}$ along diffusion path matches very well with the dependence of $D$ on $\sigma_{g g}$ (Figure 5). These results clearly indicate that force only at the window is not responsible for the existence of anomalous diffusion. It is the total force which has significant effect on the diffusion process. Unless diffusing particle size is larger than window free diameter, bottleneck associated with zeolite does not have any effect on diffusion.

We also compute average residence time, $\tau_{r}$ near window and cage between two successive intercage jumps.
Table 2 separately lists window and cage residence time. We observe excellent agreement between $D$ and $\tau_{r}$. For $\sigma_{g g}=4.96 \AA$, where diffusion minimum is seen, $\tau_{r}$ is maximum and major contribution is from the cage residence time. This is expected from Figure 10, as energy barrier in $U_{g h}$ is maximum between the window and corresponding $\alpha$-cage center. In the anomalous regime, that is $4.96 \AA<\sigma_{g g} \leq 6.0 \AA$, residence time decreases and minimum for $\sigma_{g g}=6.0 \AA$ where diffusion maximum is seen. When $\sigma_{g g}<6.0 \AA$, window residence time is negligible as compared to cage residence time suggesting no energy barrier near window during intercage diffusion. In the anomalous regime, due to existence of potential well (Figure 10) near window we observe enhancement of window residence time though it is significantly lower than cage residence time. But for $\sigma_{g g} \geq 6.3 \AA$, when $\sigma_{g g}$ is greater than window free diameter, window residence time is larger than cage residence time and for $\sigma_{g g}=$ $6.8 \AA$ window residence time is significantly larger than cage residence time suggesting bigger particle has more preference to reside near window than $\alpha$-cage. We also compute rate of intercage jump, $k_{c}$ through zeolite window and rate of cage visit, $k_{v}$. More details about $k_{c}$ and $k_{v}$ are discussed elsewhere. ${ }^{18}$ Self-diffusivity is directly related to $k_{v}$ whereas $k_{c}$ only gives how frequently a guest particle crosses the window. Table 2 lists $k_{c}$ and $k_{v}$ as a function of $\sigma_{g g}$. It is seen that even for $\sigma_{g g}=$ $6.8 \AA, k_{c}$ is much higher than the smallest size guest. This indicates that bigger size guest can cross plane of a zeolite window and perform only to and fro motion around that window.

\section{Conclusions}

Molecular dynamic simulations in the microcanonical ensemble are carried out to examine the central assumption that appearance of anomalous diffusion in 
microporous materials is due to cancellation of forces at the bottleneck. We observe that window which is the bottleneck for diffusion in macroporous materials does not play any significant role in the diffusion process unless diffusing particle size is larger than the window free diameter. Cancellation of force at the window is not responsible for the existence of anomalous diffusion regime. It is the total force which has significant effect on the diffusion process. Unless diffusing particle size is larger than window free diameter, window associated with zeolite structures does not have any effect on diffusion. Conventional wisdom is that window of a microporous material is the bottleneck for diffusion. Our study reveals that bottleneck for diffusion is always 2.6 to $3.0 \AA$ from the window and depending on diffusing particle size, it lies in different parts of the inner surface of $\alpha$-cage.

\section{Acknowledgements}

A. Sharma thanks Council of Scientific and Industrial Research (CSIR), New Delhi for funding. PKG would like to thank CSIR grant no. 01(2558)/12/EMR-II for financial support.

\section{References}

1. Tomita T, Nakayama K and Sakai H 2004 Gas seperation characteristics of ddr type zeolite membrane Microporous Mesoporous Mater. 6871

2. Kusakabe K, Kuroda T, Murata A and Morooka S 1997 Formation of a y-type zeolite membrane on a porous $\alpha$ alumina tube for gas seperation Ind. Eng. Chem. Res. 36 649

3. Schwochow W and Puppe L 1975 Zeolites and clay minerals as sorbents and molecular sieves Angew. Chem. Int. Ed. Engl. 14620

4. Holdrich W, Hesse M and Naumann F 1988 Zeolites and clay minerals as sorbents and molecular sieves Angew. Chem. Int. Ed. Engl. 27226

5. Kaduk J A and Faber J 1995 Crystal structure of zeolite $\mathrm{y}$ as a function of ion exchange Rigaku. J. 1214

6. Tanabe K and Holderich W F 1999 Industrial application of solid acid-base catalyst Appl. Catal., A 181399

7. Krishna R 2012 Diffusion in porous crystalline meterials Chem. Soc. Rev. 413099

8. Young L B, Butter S A and Kaeding W W 1982 Shape selective reactions with zeolite catalysts: Iii. selectivity in xylene isomerization, toluene-methanol alkylation, and toluene disproportionation over zsm-5 zeolite catalysts J. Catal. 76418

9. Pavelic K, Hadzija M, Bedrica L, Pavelic J, Dikic I, Katic M, Kralj M, Bosnar M H, Kapitanovic S, Blazi M P, Krizanac S, Stojkovic R, Jurin M, Subotic B and Colic M 2001 Natural zeolite clinoptilolite: New adjuvant in anticancer therapy J. Mol. Med. $\mathbf{7 8} 708$
10. Zarkovic N, Zarkovic K, Kralj M, Borovic S, Sabolovic S, Blazi M P, Cipak A and Pavelic K 2003 Anticancer and antioxidative effecs of micronized zeolite clinoptilolite Anticancer Res. 231589

11. Yang Q, Wang S, Fan P, Wang L, Di Y, Lin K and Xiao F $\mathrm{S}$ Ph-responsive carrier system based on carboxylic acid modified mesoporous silica and polyelectrolyte for drug delivery Chem. Matter. 245999

12. Bergh J V, Ban S, Vlugt T J H and Kapteijn F 2009 Modeling the loading dependency of diffusion in zeolites: The relevant site model J. Phys. Chem. C 11317840

13. Sant M, Leyssale J M, Papadopoulos G K and Theodorou D N 2009 Molecular dynamics of carbon dioxide, methane and their mixtures in a zeolite possessing two independent pore networks as revealed by computer simulations J. Phys. Chem. B 11313761

14. Jee S E and Sholl D S 2009 Carbon dioxide and methane transport in ddr zeolite: Insights from molecular simulations into carbon dioxide separations in small pore zeolites J. Am. Chem. Soc. 1317896

15. Newsman J M 1986 The zeolite cage structure Science 2311093

16. Demontis P, Gonzalez J G, Suffritti G B and Tilocca A Statics and dynamics of ethane molecules in alpo4-5: a molecular dynamics simulation study J. Am. Chem. Soc. 1235096

17. Jobic H, Karger J and Bee M 1999 Simultaneous measurement of self- and transport diffusivities in zeolites Phys. Rev. Lett. 824260

18. Yashonath S and Santikary P 1994 Diffusion of sorbates in zeolite y and a: Novel dependence on sorbate size and strength of sorbate-zeolite interaction J. Phys. Chem. 98 6368

19. Borah B J, Jobic H and Yashonath S 2009 Neutron scattering and molecular dynamics evidence for levitation effect in nanopores J. Phys. Chem. B 11312635

20. Jobic H, Borah B J and Yashonath S 2010 Levitation effect in zeolites: Quasielastic neutron scattering and molecular dynamics study of pentane isomers in zeolite nay J. Chem. Phys. 132144507

21. Yashonath S and Santikary P 1993 Influence of nongeometrical factors on the intracrystalline diffusion: importance of the sorbate-zeolite interactions Mol. Phys. 781

22. Bandyopadhyay S and Yashonath S 1995 Diffusion anomaly in silicalite and vpi-5 from molecular dynamics simulations J. Phys. Chem. 994286

23. Rajappa C and Yashonath S 1999 Levitation effect and its relationship with the underlying potential energy landscape J. Chem. Phys. 1105960

24. Jobic H, Fitch A N and Renouprez A 1986 Localization of benzene in sodium-y-zeolite by powder neutron diffraction J. Phys. Chem. 901311

25. Allen M P and Tildesley D J 1987 In Computer Simulation of Liquids (Oxford: Clarendon Press)

26. Rajappa C and Yashonath S 1997 Temperature dependence of the levitation effect implications for separation of multicomponent mixtures Faraday Discuss. 106105

27. Bandyopadhyay S and Yashonath S 1995 Diffusion anomalyin silicalite and vpi-5 from molecular dynamics simulations J. Phys. Chem. 994286 